\title{
El eterno retorno de la Asabiyyah. Ibn Jaldún y la teología política contemporánea
}

\author{
The eternal return of the Asabiyyah. Ibn Khaldun and \\ contemporary political theology
}

GIOVANNI PATRIARCA*

\begin{abstract}
Resumen: Los profundos cambios geopolíticos de las últimas décadas, el papel destabilizador de las "primaveras árabes", el proceso en curso de reestructuración del Oriente Medio han desviado la atención general hacia el pensamiento político islámico. En un contexto de crisis y desorientación, el concepto de la asabiyyah y una lectura contemporánea de la filosofía de Ibn Jaldún son fundamentales en el nombre de una interpretación moderna de la teología política clásica.

Palabras claves: Ciencia política, Historia de la filosofía islámica, Historia del pensamiento económico, Filosofía de la historia, Filosofía comparada, Teología política.
\end{abstract}

\begin{abstract}
The geopolitical changes of recent decades, the consequences of the "Arab Spring", the ongoing process of rearrangement in the Middle East have diverted public attention towards Islamic political thought. In a context of crisis and confusion, the concept of asabiyyah and a contemporary reading of the philosophy of Ibn Khaldun play a significant role in the name of a modern interpretation of classical political theology.

Keywords: Political Science, Islamic Philosophy, History of Economic Thought, Comparative Philosophy, Political Theology.
\end{abstract}

\section{Introducción}

El valor ambivalente de las "primaveras árabes" — con su papel desestabilizador en todo el mundo islámico ${ }^{1}$ - ha dado vida a procesos de reforma y contrarreforma, provocando una serie interminable de luchas internas en el intento de una "reformulación democrática" de la sociedad ${ }^{2}$. Las doctrinas políticas modernas - injertadas en ciertas experiencias culturales y en antiguas tradiciones - han dado lugar a una evolución heterodoxa pero no es un caso aislado que, en tiempos de profundo malestar, una idealizada meta-historización de

Recibido: 21/01/2017. Aceptado: 15/06/2018.

* Giovanni Patriarca ha estudiado Ciencias Políticas en la Università di Camerino (Italia) y Filosofía en la Pontificia Universidad Lateranense (Ciudad del Vaticano). Ha obtenido el Diploma de Estudios Islámicos en el Pontificio Instituto de Estudios Árabes e Islámicos-P.I.S.A.I. y el Doctorado en Filosofía en el Pontificio Ateneo Regina Apostolorum de Roma.

1 Cfr Haddad - Bsheer -Abu Rish (2012).

2 Cfr Fraihat (2016). 
un "pasado glorioso" parezca actuar como hilo conductor en un camino de confrontación y distinción radical ${ }^{3}$. Estas expectativas - con su carga emocional de transformación social y sus diferentes repercusiones en cada país - son comunes a muchas poblaciones que padecen el yugo de los problemas económicos agravados por la crisis financiera global y las altas tasas de desempleo juvenil ${ }^{4}$.

En las últimas decadas, además, los nuevos órdenes geopolíticos, generados por la implosión del bloque soviético, han cambiado las perspectivas de referencia privando al "socialismo árabe"5 - y a los movimientos laicos y anticolonialistas en él inspirados - de aquella matriz ideológica de donde recibían savia, alineación y justificación. Este proceso ha contribuido a "remodelar" los equilibrios ideológicos, aun en las peculiaridades de las diversas experiencias regionales, desviando el enfoque especulativo hacia una vía árabe-islámica, ya sea en el campo de la investigación histórica, como en el análisis político a través de una reflexión filosófica autóctona ${ }^{6}$, hija - sin duda alguna - de la tradición medieval ${ }^{7}$ y no ajena a los fundamentos de la antropología religiosa ${ }^{8}$.

En un contexto de tal índole - marcado por el desorden y aparentemente huérfano de la fascinación liberal-laicista ${ }^{9}$ - una progenitura intelectual ${ }^{10}$ y la prioridad de una autonomía interpretativa se tornan imprescindibles. Ambas arraigadas en antiguas usanzas y comprensibles para las grandes masas desorientadas, parecen ser el Leitmotiv del redescubrimiento de autores clásicos ${ }^{11}$, interpretados y glosados - no sin algunos matices ideológicos ${ }^{12}-$ a la luz de las reivindicaciones actuales ${ }^{13}$.

$\mathrm{Si}$, por un lado, este "retorno a los orígenes" ha fortalecido los lazos con la sharial4, por el otro, ha permitido - indudablemente - una cierta renovación en la interpretación de

3 “A characteristic shared by many Muslim reformists is a perceived need to return to Islam's key-sources of the Qu'ran and the tradition of the Prophet (Sunnah). In doing so they advocate a strict adherence to the literal text of the holy book and actual practices of Muhammad and his companions [...]." Olsson - Kersten (2016), 2.

4 Mabrouk (2011), 625-635.

5 "The fall of the Soviet Union was a decisive event for socialist forces throughout the world and certainly Arab Socialism is no exception. Much of the political discourse of Arab nationalism, socialism, and Marxism, with its focus in social and economic justice, "popular" democracy, the revolutionary party, and Frontal politics, has given way to a more "liberal" discourse of pluralism, human rights and civil society. Individuals and movements identified as socialist have been in power and "lost" over the course of the last several decades: the socialists have lost much of whatever claim they may have once had to represent the masses, and the people Arab socialists claim to represent have changed as well.” Browers (2006), 157-158.

6 Alatas (2006b), 29-39.

7 Marmura (2005), 18.

8 "Islam knows no distinction between a spiritual and a temporal realm, between religious and secular activities. Both realms form a unity under the all-embracing authority of the Shari' $a$. L. Massignon's definition of Islam as "une théocratie laïque égalitaire" correctly expresses this idea of Allah's sovereign law revealed through his messenger, the Prophet Muhammad, to mankind. Spiritual and temporal are the two complementary sides of the religious law." Rosenthal (1958), 8.

9 Cfr Hashemi (2009), (de especial interés el capitulo III: "Secularism and its Discontents in Muslim Societies: Indigenizing the Separation between Religion and State") 133-170.

10 Black (2001), 9.

11 Léase Lambton (1981), XVI.

12 Iskauskaite (2012), 105-146.

13 Hefner (2011), 1.

14 Crone (2005), 51. 
los autores y de las fuentes clásicas como parte de una renovada "teología política"15. Ante este panorama se entiende por qué el pensamiento de Ibn Jaldún (Tunez 1332- El Cairo 1406) está en el centro de un proceso exegético y hermenéutico ${ }^{16}$, destinado a hacer de sus ideas un punto de referencia fundamental, radicalmente independiente del canon académico occidental y de sus lecturas de la historia ${ }^{17}$.

No es una coincidencia que los círculos culturales árabes - especialmente desde el período de la descolonización de los territorios del África del Norte hasta nuestros díashayan visto en él un estudioso metódico, que - analizando críticamente los orígenes antiguos de aquellas tierras - todavía es capaz de ofrecer algunas respuestas convincentes a las presentes dificultades y retos ${ }^{18}$. Sus doctrinas se presentan ahora, a escala global, como la panacea a las vicisitudes contemporáneas del mundo islámico ${ }^{19}$, no sin generalizaciones parciales o excesivas, tanto por parte de expertos occidentales, como de intelectuales locales ${ }^{20}$.

A una cierta tradición de la historiografía occidental que reconoce su valor intrínseco, se suma el velo de genio incomprendido que caracterizó, al menos hasta hace poco, todo el trabajo científico de la obra de Ibn Jaldún. Su método profundamente analítico y riguroso $^{21}$, se muestra a los ojos de los lectores occidentales ${ }^{22}$ como un precursor de las teorías positivistas de la historia con el fin de exagerar su retrato, como ídolo ante-litteram del materialismo histórico.

Algunas tendencias actuales lo consideran, en cambio, como un antecesor del liberalismo clásico por sus críticas a la omnipotencia del poder estatal, la denuncia contra la elevada presión fiscal ${ }^{23}$ y la exaltación de la libertad ${ }^{24}$ con rasgos típicos del maquiavelismo poste-

15 Kurzman (2012), 377-390.

16 "Therefore, the generation of a modern Khaldunian sociology requires assimilating to his theory concepts and theories of the modern social sciences. But, there have been few interested in undertaking this task. This would require going beyond merely comparing some concepts in Ibn Khaldun's works with those of the modern social sciences. What is needed are systematic attempts to integrate his theory into approaches of the modern social sciences." Alatas (2006a), 407.

17 Asante (2011), 27.

18 "Islamism is seen by some scholars as a reaction to modernism, Westernization, and industrialization, and therefore they argue that Islamism is a new phenomenon. By examining the views of Islamists from centuries ago - something contemporary Islamist thinkers have certainly done- we see, however, that this movement is a new wave in an old continuum. Thus, contemporary Islamism is not a new phenomenon but one that has deep roots in the writings of traditional Islamists and in the experiences that both traditionalists and Muslim communities have encountered throughout history. There are also, some significant differences. The point of departure between traditional Islamists and new Islamists is that the former often called for total obedience to authority in order to avoid fitna (strife). However, for the latter, authority is often perceived to be un-Islamic and so they view disobedience as a justifiable form of struggle against tyranny, despite the possible resulting strife." Kirmanj (2008), 79-80.

19 "The Muslim societies confront today the challenges of a post-modern society. The crucial question is whether these countries can combine their religion with the two key sets of institutions of postmodernity: the market economy and the rule of law or human rights." Lane - Redissi (2009), 245.

20 Cfr. Zelkina (2011), 376.

21 "He (Ibn Khaldun) has conceived and formulated a philosophy of history which is undoubtedly the greatest work of its kind that has ever been created by any mind in any time and place", Toynbee (1956), 372.

22 Véase Said (1978).

23 Escartín González.-Velasco Morente.-González Abril (2012).

24 Cfr. Ahmad (1996), 6-8. 
rior $^{25}$. Aun así no parece apropriado aceptar sin reservas una u otra interpretación externa porque reducir toda su filosofía a pura "ciencia humana" — privada de cualquier herencia espiritual mediante la retórica de la secularización ${ }^{26}$ - es desatender la tradición coránica y la experiencia religiosa.

\section{El criterio de investigación y la ciclicidad de las edades}

Según Ibn Jaldún la investigación está basada en un presupuesto de conformidad con la realidad (kanun al-mutabaka). En esta perspectiva, está claro su esfuerzo por entender el motivo de la evolución de la historia embarcándose en una incesante búsqueda etiológica de las "leyes sociales"27. Como condición indispensable para el estudio de la historia se requiere por un lado que esta ciencia sea independiente ('ilm bi-mustakill nafsih) y por otro que tenga como primer objeto de análisis la civilización humana (al-'umran al-bashari), entendida como el conjunto de sus características generales ${ }^{28}$.

En su doctrina, el contexto geográfico y ambiental está profundamente ligado a un ideal estrictamente antropológico, en el cual se desarrolla de manera original un concepto de las generaciones con un examen tan moderno de las variables sociales que el lector contemporáneo se queda positivamente asombrado y sorprendido. Su estructura de la civilización ofrece un espectro de formas institucionales y de leyes comunes generales - aplicables mutatis mutandis a diferentes edades y épocas - en una exégesis del espíritu y del destino de los pueblos.

En efecto, en los seis capítulos de los Muqaddima ${ }^{29}$ se parte de un estudio de la influencia socio-antropológica del medio ambiente sobre la naturaleza humana, para luego hacer una descripción de las primeras civilizaciones "nómadas" ( "umran badawi). El concepto de 'Umran abarca una multiplicidad de significados: desde un lugar geográfico habitado hasta una sociedad stricto et latu sensu ${ }^{30}$. A continuación, el foco de interés se desplaza desde las primeras formas institucionales y sus características evolutivas hasta las formas más desarrolladas y sofisticadas de agregación social en un contexto urbano y sedentario (hadari 'umran). De esta manera se llega a analizar los modos de comercialización y desarrollo de

25 Morris (2009), 242.

26 "In contrast to the West, the respective realms of religion and state are intimately intertwined in Islam and subject to a process of fluid negotiation; the concepts of authority and duty overshadow those of freedom and the rights of individual. Islamic political thought not only deals with matters of government, politics, and the state but also addresses questions of acceptable behavior and ethics of both the ruler and the ruled before God. Islamic political thought cannot be measured by Western criteria and standards of political theory. It must be understood from within its own tradition, characterized by a vibrant integration of the secular and sacred in obedience to God and His Prophet. In its very nature, Islam is dynamic, not static, both as a way of life and as a way of monotheistic worship. It is a living reality rather than a frozen sytem.” Böwering- Crone - Mirza (eds.) (2013), Introduction - VIII.

27 Vivanco Saavedra (2000), 27-43.

28 Cfr Patriarca (2013a), 352-363.

29 Ibn Khaldun (1993).

30 Cfr Lacoste (1984), 93. 
las actividades de fabricación y su inherente contribución al florecimiento de una sociedad venturosa donde progresan - libremente y sin obstrucción - filosofía, cultura y arte ${ }^{31}$.

El complejo órganon metodológico refleja una dinámica histórica en la cual aparecen, en palabras modernas, los Idealtypen ${ }^{32}$ sociales al analizar dicotomías estructurales como la ciudad/el campo, la sociedad nómada/sedentaria, la solidaridad tribal/el egoísmo individualista ${ }^{33}$. Esta visión global de la historia permite formular una teoría caracterizada por ciclos económicos en los cuales el Estado (primera y segunda generación), originalmente, tiene un poder limitado y los impuestos están en un nivel relativamente bajo para permitir un crecimiento sostenido de la producción y del consumo ${ }^{34}$. Después de estas fases, (tercera generación) el Estado concentra sobre sí mismo todo el poder, mediante el aumento de la carga fiscal y la reducción de la libertad de iniciativa seguida por una clara disminución en el consumo y en la producción ${ }^{35}$. La fase final (cuarta generación) consiste en un fuerte estancamiento económico cuyo principal efecto es la parálisis del Estado en su conjunto.

Los primeros síntomas de esta deslegitimación del poder original están, de hecho, estrechamente vinculados a las políticas económicas y a la regulación fiscal ${ }^{36}$. Sin el estímulo personal para la productividad y el compromiso, la sociedad está condenada a auto-excluirse en la apatía causada por un sistema político que es coercitivo y carente de respeto hacia la libertad individual. Ibn Jaldún afirma en una famosa conjetura que "en el comienzo de una dinastía, la tributación general obtiene un grande ingreso de pequeños impuestos. Al final de una dinastía, la tributación obtiene un pequeño ingreso de grandes impuestos." ${ }^{37}$

En esta anticipación precisa de la curva de Laffer ${ }^{38}$, Ibn Jaldún explica muy claramente que después de haber perdido sus hábitos austeros y habiendo aumentado sus necesidades de lujo $^{39}$, los soberanos imponen una pesada carga tributaria en sus súbditos, elevando el nivel de la vieja fiscalidad para ampliar sus beneficios ${ }^{40}$. El efecto de este aumento es la radical

31 "Es decir, para Ibn Jaldún el tema del conocimiento es clave para explicar la vida social. Los conocimientos acumulados y aplicados a la vida cotidiana es fuente de progreso y bienestar. Pero tales conocimientos no se improvisan. Pueden aprenderse, transmitirse. Pero necesitan de unos hábitos adquiridos (una rutinas asumidas y vividas durante generaciones) y unas condiciones contextuales adecuadas, que permitan que tales hábitos puedan arraigarse, y convertirse en una segunda naturaleza para los habitantes de un determinado lugar. No se trata, por tanto, de la existencia de individualidades relevantes. Sino de la asunción social de determinados patrones. En la medida en que la creatividad se convierte en hábito, en rutina generalizada, el avance técnico será posible. En la medida en que el hábito sea la repetición de modos y soluciones, difícilmente se saldrá de los moldes del pasado. Pero las condiciones son fundamentales para que puedan aparecer unos hábitos u otros. Por supuesto en términos estrictos de oferta y demanda suficiente, para que se forme un espacio económico adecuado ("el provecho que pudiera reportar" es, para nuestro autor, un incentivo incluso para la propia creatividad científica). Pero también, en términos de estabilidad institucional y política. Todo lo que implique inestabilidad, falta de referentes claros, modificación sistemática del marco jurídico..., dificulta la constitución de hábitos adecuados.", García Lizana (2006), 173.

32 Cfr Weber (1904).

33 Baeck (1994), 115.

34 Ibn Khaldun, Muqaddima, vol. 2:103-104. (Traducción nuestra)

35 Ibn Khaldun, Muqaddima, vol. 1:106.

36 Andic - Andic (1985), 454-469.

37 Ibn Khaldun, Muqaddima, vol. 1:89 (Traducción nuestra).

38 Gwartney - Stroup - Sobel - Macpherson (2008), 100.

39 Cfr Ibraham (1988), 51-57.

40 Cfr Boulakia (1971), 1105-18. 
disminución de la actividad económica tanto que, en esta delicada situación, el "gobierno" parece no tener otra solución que una depreciación de la moneda generando una serie infinita de males como en profundidad demostró Al-Makrizi, distinguido discípulo egipcio de Ibn Jaldún, en sus obras sucesivas ${ }^{41}$.

\section{Asabiyyahh como motor de la historia}

En esta teoría de la evolución y decadencia — en la que son analizados todos los síntomas y los males por los cuales una sociedad nace, crece y muere - juega un papel fundamental la asabiyya $^{42}$. Admirablemente descrita por Ibn Jaldún, es un concepto desconocido en el mundo occidental cuyos aspectos fundamentales muchos estudiosos han tratado de aclarar en el cursos de los años, a veces con artificios retóricos, para lograr una interpretación más clara $^{43}$.

El concepto alude a - según las diferentes definiciones de los historiadores del pensamiento jalduniano ${ }^{44}$ - la " mutua solidaridad", la "cohesión de grupo", la "voluntad común", el "espíritu de coalición", la "Lebenskraft" ". Se trata de un impulso visceral de pertenencia y de un vínculo metapolítico ${ }^{46}$ que reúne y fortalece a todos los miembros de la comunidad, formando un cuerpo ideal ${ }^{47}$ unido en un esfuerzo compartido para lograr el objetivo común de la convivencia y del orden ${ }^{48}$. Este "esprit de corps" se levanta como una barrera protectora ${ }^{49}$ en defensa de la colectividad desde una perspectiva intergeneracional de continua palingenesia ${ }^{50}$. A este Volksstreben está ligada la suerte de la sociedad porque la asabiyyah es "die motorische Kraft im staatlichen Geschehen." 1

En la parábola histórica de cada civilización se puede ver "el desarrollo, el clímax y el desgaste" ${ }^{52}$ de estos lazos de solidaridad que han sido su fuerza inherente ${ }^{53}$. Para fortalecer el espíritu y el poder político se necesita una creencia religiosa como común referncia de

41 Ashtor (1977), 101-110.

42 Cfr Fischel (1967).

43 Colosio (1914), 308-338.

44 Véase Campanini (ed.) (2005).

45 "Jede dieser Definitionen birgt in sich eine Teilwahrheit, aber, um die ursprüngliche Bedeutung in ihrer vollen Tiefe zu verstehen, darf man nicht nach Maßstab der säkularen Mentalität eine Aufteilung vornehmen, weder glatt noch künstlich, von einer starken religiösen Unterschicht, welche diesen nuancenreichen Begriff nicht selten in ein Mystisches Licht rückt. Eine innige Gegenseitigkeit bindet jedes Mitglied der Gemeinschaft in eine solidarische Kraft, ausgerichtet auf ein Gemeinschaftsziel.” Patriarca (2011).

46 Bianchin (2006), 25.

47 Cfr. Cavarero (1995), 113-114.

48 Léase Gabrieli (1930), 473-512.

49 Léase Riedel (1975).

50 Cfr. Suurland (2009), 270.

51 "La fuerza motor del devenir estatal". Rosenthal (1932), 57.

52 Noja, S. (1997), 155 (Traducción nuestra).

53 "At the very beginning of modernization process, societies are traditional, religious, and pre-modern; but at the same time they are "active" societies with functioning neighborhood structures, and very clear perception of what are "good" and "evil", and a high respect of the law. As the modernization process proceeds, traditional religious values seem to be on the losing side of the societal equation." Tausch -Heshmati (2009). 
conducta. "Una dinastía - afirma Ibn Jaldún - que comienza su historia basándose en la religión, duplica la fuerza de la asabiyyah que la ayuda en su formación."54

En cuanto a su concepción del desarrollo social, Ibn Jaldún declara repetidamente que no es la cantidad de la reserva de moneda o de metal precioso la medida justa de la prosperidad de un país, sino la mayor especialización y división del trabajo entre sus habitantes. Esto, de hecho, crea un "círculo virtuoso" que aumenta la productividad en una justa distribución de funciones y riesgos. Las autoridades tienen el derecho a una remuneración por los servicios prestados para la protección de la propiedad pública y privada, no interfiriendo en otras actividades ${ }^{55}$.

Según Ibn Jaldún, anticipando también a las teorías sociológicas de la urbanización, las ciudades son el caleidoscopio de la vitalidad de una sociedad: cuanto mayor es la presencia de personas, mayor es la especialización en la cooperación social recíproca, porque nadie es autosuficiente. A la prosperidad y a la riqueza de la población sigue un gran aumento demográfico que genera procesos adicionales de producción y consumo. Pero, al mismo tiempo, en la ciudad se encuentra el germen venenoso del abandono a la lujuria ${ }^{56}$ y se lleva a cabo aquel proceso inversamente proporcional que destruye la civilización con sus mismas $\operatorname{armas}^{57}$. El lujo y los vicios son la causa-raíz del debilitamiento de aquellas íntimas obligaciones de respaldo y respeto sobre las cuales la sociedad ha puesto sus bases y gracias a las cuales debe su supervivencia.

\section{Desarrollo contemporáneo y sus consecuencias}

El cíclico y regular redescubrimiento del pensamiento social de Ibn Jaldún ${ }^{58}$ en tiempos de dificultades y envilecimiento está sujeto a fuertes contrastes y se mezcla con iniciativas políticamente sesgadas como ya sucedió, en parte, durante el período de descolonización y con el nacimiento de formas extremas de nacionalismo ${ }^{59}$.

Se percibe también una interpretación - sub specie modernitatis - de matriz fundamentalista que reúne - con propósitos puramente propagandísticos - diferentes escuelas de pensamiento que sin embargo, durante siglos, se han opuesto o han sido una fuente de

54 Ibn Khaldun, Muqaddima, vol. 2:325

55 Véase Cumsille (2011), 173-205.

56 "His emphasis on luxury as the main cause of a civilization's decline may be seen as an overexaggeration, even though he observed the decline of several states that enjoyed luxury. Moreover, the characteristics he attributed to urbanites, e.g., "immorality", "wrongdoing", "insecurity", "trickery", and devotion to "lying", "gambling", "cheating", "fraud", and "theft", constitute a sweeping generalization. Not all urbanites, including Ibn Khaldun himself, have to devote themselves to these types of deviant behavior. The association between luxury and the creation of these forms of deviant behaviors is neither clear nor convincing. Only after the association between the decline of religion as a form of social control and the increase of deviance in urban areas was made did Ibn Khaldun provide a convincing explanation.” Baali (1988), 89.

57 "The preconditions of civilization and of cohesion are mutually antithetical. These two plants will not grow in the same soil. The society he knew was neither a purely tribal one, such as could do without civilization, nor a properly town-dominated one, such as could do without tribal cohesion. Each of two elements was ever-present and essential. The story of the interplay of these two elements, of these repetitive stalemate position, is at the core of Ibn Khaldun's vision." Gellner (1995), 88.

58 Cfr. Al-Azmeh (2003).

59 Véase Carré (2004). 
mutuo resentimiento ${ }^{60}$, todo en nombre de una rediviva pax theologica de expressión sunní61. En este mosaico confuso e incierto aparece aquella perenne tensión entre las aspiraciones de un retorno a una época gloriosa, con contornos claramente meta-históricos y tintes profundamente escatológicos ${ }^{62}$ y los intentos de modernización interpretativa ${ }^{63}$, a menudo impopulares o descaradamente contrapuestos ${ }^{64}$.

A raíz de los últimos acaecimientos, se muestra más actual que nunca una pregunta retórica del filósofo orientalista H. Corbin, que en una de sus obras más conocidas, no duda en afirmar:

En resumen, después de la desaparición de Averroes, ¿fue Ibn Arabî o fue Ibn Khaldûn quien indicó al Islam espiritual su auténtica finalidad? La crisis radical de nuestros días, ¿tiene como causa remota el hecho de que Ibn Khaldûn no tuvo seguidores? ¿O bien se debe, por el contrario, a que la secularización moderna, al cumplir las previsiones de Ibn Khaldûn, ha ido acompañada de la desaparición de lo que ha representado y representa aún un Ibn Arabî? Tanto en un caso como en otro, se le plantea al filósofo la cuestión de saber si la reducción de las «ciencias divinas» a las «ciencias humanas» responde al destino del hombre. No es sólo la suerte de la filosofía islámica la que está en juego, sino la vocación misma del Islam en este mundo, la validez del testimonio que el Islam da en este mundo desde hace catorce siglos. La grandeza, trágica sin duda, de Ibn Khaldûn está para nosotros en el hecho de que conduce a la conciencia a delimitar estas cuestiones. ${ }^{65}$

Se asiste, en cualquier caso, a un proceso de extrema "teologización" de Ibn Jaldún, tanto que, en este sentido, un artículo crítico escrito por J. Khaki afirma expresamente que la historia, por desgracia, en muchos casos recientes, especialmente en aquellos países donde hay una reformulación y re-estructuración de los contrapesos políticos, se considera "una herramienta para justificar posiciones sectarias e ideológicas o para difamar a otras. El objetivo no es encontrar la verdad en la historia sino usar la historia para justificar las posiciones teológicas que se basan sobre hipótesis e interpretaciones de la historia, que podrían denominarse historias teologizadas o ideologizadas"66.

60 Léase Hentati (2007) 46-47.

61 Cfr Winkel (1995) 108-110.

62 Cfr Cook (2002) 209-215.

63 Métraux (ed.) (1977), 109.

64 Taji-Farouki (ed.) (2006).

65 Corbin (2000), 547.

66 "Exceptions apart, many history books written in Muslim countries, [...], methodologically, structurally, and content-wise leave much to be desired. Some history books are even sprinkled with curses and abuses for personalities whom the writers did not like or approve of. Such is the sad story of some of the writers of our history in many Muslim societies. One reason why this tendency seems to have developed is that history is approached as a tool to justify sectarian or ideological positions and defame others. The purpose is not finding the truth in history but using history to justify theological positions based on their assumptions and interpretations of history, which might be called 'theologised' or 'ideologised' histories. Many other nations also tend to do this in the name of nation-building, which may be called nationalised histories. As opposed to this phenomenon, one can find versions of history written by many Muslim writers who have tried to investigate history from a more objective position trying to draw so-called scientific conclusions. One such marvelous example is that of 
Este aspecto no es nuevo en la historia universal y va ligado a la naturaleza humana. Sin embargo, frente a una amalgama que agrupa las más diversas exigencias políticas, económicas y sociales sobre la base de una lectura estrictamente inspirada en la ley divina, aparecen visiblemente - sin ningún "filtro diplomático"- los ya usuales sentimientos de oposición a todo lo que es ajeno a dicha tradición. Aún cuando la reflexión filosófica de Ibn Jaldún hace eco de los temas clásicos de la teología musulmana ${ }^{67}$ y de las disputas jurídicas sobre las formas de violencia ${ }^{68}$, sobre la licitud de la coerción ${ }^{69}$ y sobre la justificación de la guerra ${ }^{70}$, no se puede reducir el valor universal de su pensamiento pragmático ${ }^{71}$ y de su enfoque epistemológico en su mera función legalista o monolítica al servicio de una visión partidista $^{72}$, intolerante $\mathrm{y}$, a veces, agresiva $^{73}$.

Algunas interpretaciones contemporáneas ${ }^{74}$, en efecto, subrayan ciertos aspectos en detrimento de otros tantos dando como resultado una "fusión", sutilmente impuesta y tal vez ilegítima $^{75}$, entre el pensamiento del filósofo de Túnez y las ideas teológicas de Ibn Taymiyya ${ }^{76}$

Ibn Khaldun. Today, Ibn Khaldun is seen as a shining example of this trend where he tries to study history not as theology, but as a science, an objective study of history without attaching any epithet. In sum, writers should use the terms 'Islamic' or 'Muslim' discerningly which can prevent standardisation of everything Muslims do or don't do in a particular society at a particular time and in a particular context.” Jan-e-Alam Khaki (2013), (Traducción nuestra).

67 Cfr. Cruz Hernández (1996), 668 y ss.

68 Véase El-Fadl (2001) y Butterworth (1990), 79-100.

69 Cfr. Khan (2006), 156.

70 "Ibn Khaldun begins his discussion of war by saying that these "have always occurred in the world since God created it", naturally and unavoidably, because of men's desire for revenge and their need for self-defense. Ibn Khaldun then identifies four types of war. The first of these "usually occurs between neighboring tribes and competing families." The second is "war caused by hostility", whereby "savage nations living in the desert" attack their neighbors, solely with a view to seizing their property. These two types are "wars of outrage and sedition" (hurub baghy wa fitna). The third type is "what the divine law calls jihad". The fourth consists of "dynastic wars against seceders and those who refuse obedience." Of these four types, "the first two are unjust and lawless", while the last two are "wars of jihad and justice" (hurub jihad wa-'adl). In this way, as Charles Butterworth remarked, Ibn Khaldun "distinguishes just war from jihad and allows neither to encompass the other." Bonner (2006), 6.

71 "La pensée de Ibn Khaldun est hostile à toute sorte de spéculation pure. L'homme est incapable de dépasser, dans et par la pensée, 1’horizon de la nature et de réalités concrètes ; ni l'abstraction systématisée, ni la causalité métaphysique ne peut garantir une connaissance sure." Nasser (1997), 90.

72 Sival, E. (1990), 94-107.

73 Choueiri (2010), 224-227.

74 Mona (2010), 318-366.

75 "Students of Ibn Taymiyya were prevalent and influential in the fourteenth century Marinid courts. In contrast to Ibn Khaldun, who was himself a sufi in many respects and had immense respect for the Sufis of rural Morocco, abd Al-Aziz al-Qayrawani (d. 1349 or 1350) saw some sufi leaders or sheikhs of shrine as potential threats to the official religious authorities, or ulama, who were more directly dependent on the sultan. In a chapter of the Muqaddimah dedicated to the methods of the Sufi saints, Ibn Khaldun called accusations against the Sufi wrongheaded and firmly defended the Sufi way against the narrow-mindness of jurists trained at official madrasas.” Fromherz (2010), 47.

76 Cfr Laoust (1939). 
(Harran 1263-Damasco 1328) y de sus discípulos ${ }^{77}$, conocidos por su convencida batalla ${ }^{78}$ a favor de la "estricta observancia" 79 en el álveo de la escuela hanbalita ${ }^{80}$ y más precisamente en su derivación salafí1 ${ }^{80}$. Se obstaculiza de este modo - casi hasta la prohibición ${ }^{81}$ - cualquier otra experiencia especulativa ${ }^{82}$ y con ello toda expresión no propiamente ortodoxa o proceso lógico-crítico ${ }^{83}$, posible fuente de hibridación cultural y disidencia ${ }^{84}$.

Llegado ya el final de este ensayo, conviene dejar constancia de cómo es evidente que la centralidad de la asabiyyah ${ }^{85}$ - con su inopinable ascendencia tracendental ${ }^{86}$ y apoyada por referencias coránicas - sobrepasa los límites de un supuesto Estado-nación ${ }^{87}$. Su importancia se ve incrementada por la condena a la fitna, la cual incorpora los conceptos de sedición, de corrupción, de escándalo y de guerra civil ${ }^{88}$.

77 "The reputation of Ibn Taymiyya and the number of his disciples thus ensured that Hanbalism was well represented in the new phase of Islamic thought brought about by this transference. Hanbalism here gained a base - or should we say a beach-head? - from which it was able to influence later centuries." Montgomery Watt (2009), 163.

78 "La sua ossessione per la "differenza", tipica di un mondo minacciato e in fase di cambiamento, ben si confà all'esasperata ricerca di identità che caratterizza parte del mondo musulmano contemporaneo.” Bori (2003), 171.

79 Krawietz (2003), 52-57.

80 Véase Makdisi (1983).

81 Al-Matroudi (2006).

82 Véase Ibn Taymiyya (1993).

83 "Pero el gran intelectual musulmán opositor al saber científico fue, sin duda, el jurista hanbalí Ibn Taymiyya (m. en 1238), el cual pensaba que todo el sistema lógico aristotélico se basaba en una doctrina metafísica que amenazaba el concepto mundial del Islam y también que las formas aristotélicas de argumentación eran hostiles a los modos islámicos de pensamiento.", Martos Quesada (2006), 82-83.

84 "Ibn Taymiyya's manner of argumentation in favour of these fixed positions was often quite original, as has been emphasized in recent scholarship, and his ability to enlist reason as an handmaiden to dogma, without allowing it to supersede textual originalism may well explain why his works has become the standard exposition of Salafi theology for later generations." Lav (2012), 42.

85 Gierer (2001), 91-104.

86 "La religión tiene que ver directamente con las peculiaridades del observar. Todo observar debe distinguir para poder señalar algo, de esa manera separa al "marked space" en donde se retira el último horizonte del mundo. La trascendencia que con ello acompaña a todo lo aprehensible se desplaza en cada entento de trascendencia está siempre presente como el otro lado de lo determinado sin poderse alcanzar jamás. Precisamente esta inaccesibilidad "liga" al oservador - quien a su vez es inalcanzable para la observación - a aquello que él puede señalar. Religar lo no-señalable en lo señalable - esto es "religio" en el más amplio de los sentidos, independientemente de sus características culturales." Luhmann (2007), 178-179.

87 "Umma beschreibt dabei die Gemeinde der Gläubigen, während "Asabiya” auf die in dem o.g. Zitat von Max Weber genannten Sippenzusammenhänge verweist. In der allgemeinen innerislamischen Rhetorik wurde die Umma stets der Asabiya vorgezogen, was aber nichts an der sozialen Bedeutung der Sippenbande und schranken in der islamischen Welt ändern konnte. Die Umma ist diejenige soziale Verbindung, die, wie die Tönnies' Gesellschaft, die verwandtschaftliche Verbindung überwindet, dabei aber den Charakter der allgemeinen Brüderlichkeit für sich in Anspruch nimmt. Dass nun die Umma keine empirische soziale Sachlage, sondern eher eine Wunschvorstellung islamischer Propaganda widerspiegelt, ist kein Einwand gegen die soziale Wirksamkeit dieses Gesellschaftsbildes. Auch die Gemeinschaft als die bessere Alternative zu Gesellschaft ist idealisiert worden." Grutzpalk (2007), 33.

88 Cfr Patriarca, G. (2013b), 541-548. 
Tal perspectiva de actualización ${ }^{89}$, articulada en diferentes grados y llena de proyectos utópicos ${ }^{90}$, encuentra terreno fértil en una realidad de cambios epocales, de marcada desorientación y de consecuente defensa identitaria.

\section{Referencias bibliográficas}

Ahmad, I.A. (1996), "Islam and Markets", Religion \& Liberty, vol. 6-n.3.

Alatas, S.F. (2006a), "A Khaldunian Exemplar for Historical Sociology for the South", Current Sociology, vol. 54, no. 3.

Alatas, S.F. (2006b), "Religion and Reform. Two Examples for Autonomous Sociology in the Non-Western Context", en S. Patel (ed.), The ISA Handbook of Diverse Sociological Traditions, SAGE Studies in International Sociology, London.

Al-Azmeh, A. (2003), Ibn Khaldun. An Essay in Reinterpretation, Central European University Press, Budapest.

Al-Matroudi, A.H.I. (2006), The Hanbali School of Law and Ibn Taymiyya. Conflict or Concilliation, Routledge, New York.

Andic, F. M. -Andic, S. (1985), "An Exploration into Fiscal Sociology: Ibn Khaldun, Schumpeter, and Public Choice", FinanzArchiv / Public Finance Analysis, New Series, Bd. 43, H. 3.

Asante, M. K. (2011), "De-Westernizing Communication. Strategies for Neutralizing Cultural Myths" en G. Wang (ed.), De-Westernizing Communication Research. Altering Questions and Changing Frameworks, Routledge, New York.

Ashtor, E. (1977), "The Development of Prices in Medieval Near East”, en B. Spuler (ed.), Geschichte der islamischen Länder. Wirtschaftsgeschichte des Vorderen Orients in islamischer Zeit, Band 6 - Teil I, E. J. Brill, Leiden.

Ashworth, L. M. (2007), "Ibn Khaldun and the Origins of the State Politics", in Persram N. (ed.) (2007), Postcolonialism and Political Theory, Lexington Books, Plymouth.

Baali, F. (1988), Society, State and Urbanism. Ibn Khaldun's Sociological Thought, State University of New York Press, Albany.

Baeck, L. (1994), The Mediterranean Tradition in Economic Thought, Routledge, London.

Black, A. (2001) The History of Islamic Political Thought: From the Prophet to the Present, Routledge, New York.

Bonner, M. (2006), Jihad in Islamic History. Doctrine and Practice, Princeton University Press, Princeton.

Bori, C. (2003), Ibn Taymiyya. Una vita esemplare. Analisi delle fonti classiche della sua biografia, Istituti Editoriali e Poligrafici Internazionali, Bari.

Boulakia, J.D. (1971), “Ibn Khaldun. A Fourteenth Century Economist.”, Journal of Political Economy 79, n. 5.

89 Léase Katsiaficas (1999) 45-57.

90 "Ahistorical utopias made no sense to Ibn Khaldun, since such a state would be divorced from the particular conditions in which it found itself. While divine truth was eternal, political truth was subject to the flux of a cyclical history. Yet, this flux of history was not without its sense of order. While the passage of time led to quite abrupt changes in the forms and fortunes of political institutions, these changes fit into certain knowable laws of history." L. M. Ashworth (2007), 44. 
Böwering, G., Crone, P. \& Mirza, M. (eds.) (2013), The Princeton Encyclopedia of Islamic Political Thought, Princeton University Press, Princeton.

Browers, M. (2006), Democracy and Civil Society in Arab Political Thought: Transcultural Possibilities, Syracuse University Press, Syracuse.

Butterworth, C. (1990), “Al-Fārābī’s Statecraft: War and the Well-Ordered Regime.” en J. Turner Johnson- J. Kelsay (eds.), Cross, Crescent, and Sword: The Justification and Limitation of War in Western and Islamic Tradition, Greenwood Press, New York.

Campanini, M. (ed.) (2005), Studies on Ibn Khaldun, Polimetrica, Monza.

Carré, O. (2004), Le Nationalisme arabe, édition Payot, Paris.

Cavarero, A. (1995), Corpo in figure. Filosofia e politica della corporeità, Feltrinelli, Milano.

Choueiri, Y.M. (2010), Islamic Foundamentalism. The Story of Islamist Movements, Continuum International Publishing, London.

Colosio, S. (1914), “Contribution à l'etude d'Ibn Khaldun”, Revue du Monde Musulman, XXVI.

Cook, D. (2002), Studies in Muslim Apocalyptic, The Darwin Press, Princeton N.J.

Corbin, H. (2000), Historia de la filosofía islámica, Editorial Trotta, Madrid.

Crone, P. (2005), Medieval Islamic Political Thought, Edinburgh University Press, Edinburgh.

Cruz Hernández, M. (1996), Historia del pensamiento en el mundo islámico, Vol. 3, Alianza Editorial, Madrid.

Cumsille, K. (2011), “Ibn Jaldún. Una teoría política del poder constituyente”, Revista Pleyade, vol. IV-n. 1

El-Fadl, K.A. (2001), Rebellion and Violence in Islamic Law, Cambridge University Press, Cambridge.

Escartín González, E.-Velasco Morente, F.-González Abril, L. (2012) “Impuestos moderados según Ibn Jaldún", Medievalista, N. 11 [sitio en internet] :

http://www2.fcsh.unl.pt/iem/medievalista/MEDIEVALISTA11/PDF11/PDFjaldun1104.pdf

Fischel, W. J. (1967), Ibn Khaldun in Egypt. His Public Functions and His Historical Research, University of California Press, Berkeley.

Fraihat, I. (2016), Unfinished Revolutions. Yemen, Libya, and Tunisia after the Arab Spring, Yale University Press, New Haven.

Fromherz, A.J. (2010), Ibn Khaldun. Life and Times, Edinburgh University Press, Edinburgh.

Gabrieli, F. (1930), "Il concetto della "asabiyyah nel pensiero storico di Ibn Haldûn", Atti della Reale Accademia delle Scienze di Torino. Classe di Scienze morali, storiche e filologiche, LXV.

García Lizana, A. (2006), "Tradición y Progreso: Las claves del futuro", Cuadernos de CC.EE. y EE. (Universidad de Málaga), n. 50-51.

Gellner, E. (1995), Muslim Society, Cambridge University Press, Cambridge.

Gierer, A. (2001), "Ibn Khaldun on Solidarity - Modern Science on Cooperativeness and Empathy: A Comparison", Philosophia Naturalis, n. 38.

Grutzpalk, J. (2007), “Umma und Asabiya: ein muslemisches Gegenkonzept zu „Gemeinschaft und Gesellschaft“ oder eine mögliche Ergänzung der Tönnieschen „Grundbegriffe 
der reinen Soziologie", Tönnies Forum-Rundbrief der Ferdinand Tönnies-Gesellschaft, v. $16 / 1$.

Gwartney, J. -Stroup, R.- Sobel, R. -Macpherson, D. (2008), Macroeconomics: Public and Private Choice, Cengage Learning, Boston.

Haddad, B. - Bsheer, R. -Abu Rish, Z. (2012), The Dawn of the Arab Springs: End of an Old Order?, Pluto Press, London.

Hashemi, N. (2009), Islam, Secularism, and Liberal Democracy: Toward a Democratic Theory for Muslim Societies, Oxford University Press, Oxford.

Hefner, R. W. (2011), Shari'a Politics: Islamic Law and Society in the Modern World, Indiana University Press, Bloomington, IN.

Hentati, N. (2007), Les écoles juridiques en Afrique du Nord. De l'origine au $V^{\circ}-X I^{\circ}$ siècle, (ed. par C. Intartaglia), Etudes Arabes n.100-101, PISAI-Pontificio Istituto di Studi Arabi e di Islamistica, Roma.

Ibn Khaldun (1993), The Muquaddima: An Introdction to History, (trans. F. Rosenthal) Princeton University Press, Princeton.

Ibn Taymiyya (1993), Against the Greek Logicians, (edited and translated by W.B. Hallaq), Clarendon Press, London.

Ibraham, H. (1988), "Leisure, Idleness and Ibn Khaldun", Leisure Studies, vol. 7, Issue 1.

Iskauskaite, R. (2012), "The Complexity of the Concept of Islamic Revivalism and Revivalists' Understanding of a Proper Model of State", Politologija, no. 2 (66), pp. 105-146.

Jan-e-Alam Khaki (2013), "Muslim vs Islamic", [sitio en internet] www.dawn.com , 18 May 2013.

Katsiaficas, G. (1999) “Ibn Khaldun: A Dialectical Philosopher for the 21st Century”, New Political Sciences, vol. 21 n.1.

Khan, A. (2006), "Islam as Intellectual Property: "My Lord! Increase me in Knowledge." en H. M. Ramadan, Understanding Islamic Law: From Classical to Contemporary, AltaMira- Rowman\&Littlefeld, Oxford.

Kirmanj, S. (2008) "'The Relationship between Traditional and Contemporary Islamist Political Thought", Middle East Review of International Affairs, no. 12 (1).

Krawietz, B. (2003), "Ibn Taymiyya, Vater des islamischen Fundamentalismus? Zur westlichen Rezeption eines mittelalterlichen Schariatsgelehrten", in M. Atienza (Hrsg), Theorie des Rechts in der Gesellschaft, Duncker\&Humblot, Berlin.

Kurzman, C. (2012), «The Arab Spring Uncoiled», Mobilization: An International Quarterly, vol. 17, no. 4, pp. 377-390.

Lacoste, Y. (1984), Ibn Khaldun. The Birth of History and the Past of the Third World, Verso Editions, London.

Lambton, A.K.S. (1981), State and Government in Medieval Islam, Oxford University Press, Oxford.

Lane, J-E. - Redissi, H. (2009), Religion and Politics: Islam and Muslim Civilization, Ashgate Publishing, Farnham.

Laoust, H. (1939), Essai sur les doctrines sociales et politiques d'Ibn Taymiyya, Institut Français d'Archéologie Orientale, Cairo.

Lav, D. (2012), Radical Islam and the Revival of Medieval Theology, Cambridge University Press, Cambridge. 
Luhmann, N. (2007), La sociedad de la sociedad, Editorial Herder-Universidad Iberoamericana, México D.F.

Mabrouk, M. (2011), “A Revolution for Dignity and Freedom: Preliminary Observations on the Social and Cultural Background to the Tunisian Revolution", Journal of North African Studies, vol. 16, issue 4, pp. 625-635.

Makdisi, G. (1983), L'Islam hanbalisant, Geuthner, Paris.

Marmura, M.E. (2005), "Medieval Islamic Philosophy and the Classic Tradition”, en J. Inglis (ed.), Medieval Philosophy and the Classical Tradition: In Islam, Judaism and Christianity, Routledge, New York.

Martos Quesada, J. (2006), "Islam y ciencia en Al-Andalus", 'Ilu. Revista de Ciencias de las Religiones, Anejo XVI.

Métraux, G.S. (ed.) (1977), Pensée et valeurs de l'Islam, Cultures, Vol. IV n.1, Les Presses de l'Unesco et la Balconnière, Paris.

Meijer R. (ed.) (2009), Global Salafism: Islam's New Religious Movement, Hurst, London

Mona, H. (2010), "Modern Interpretations and Misinterpretations of a Medieval Scholar: Apprehending the Political Thought of Ibn Taymiyya", en Y. Rappaport (ed.), Ibn Taymiyya and his Times, Oxford University Press, Oxford.

Montgomery Watt, W. (2009) Islamic Philosophy and Theology, Aldine Transaction, Pescataway-N.J. 2009.

Morris, J. W. (2009), “An Arab Machiavelli? Rhetoric, Philosophy and Politics in Ibn Khaldun's Critique of Sufism", Harvard Middle Eastern and Islamic Review, n. 8.

Nasser, N. (1997), La pensée réaliste d'Ibn Khaldun, Presse Universitaire de France, Paris.

Noja, S. (1997), Breve storia dei popoli dell'Islam, A. Mondadori, Milano.

Olsson S.- Kersten C. (2016), Alternative Islamic Discourses and Religious Authority Contemporary Thought in the Islamic World, Routledge, New York.

Patriarca, G. (2013a), "A Medieval Approach to Social Sciences. The Philosphy of Ibn Khaldun. Some Historical Notes and Actual Reflections”, en S. Alvi- A. Al-Roubaie (eds.), Islamic Economics. Critical Concepts in Economics, Vol. I, Part IV, n. 21. Routledge, New York.

Patriarca, G. (2013b), “Il caleidoscopio dell'assabyia: analisi, rischi e prospettive. L'attualità di Ibn Khaldun in un contesto di crisi”, Studia Politica - Romanian Political Science Review, vol. XIII, n. 3.

Patriarca, G. (2011), "Die Rückkehr der Assabiya", [sitio en internet] Al-Sharq, disponible en www.alsharq.de/2011/hintergrund/die-ruckkehr-der-assabiya- , acceso el 27 de mayo de 2011.

Riedel, M. (1975), Metaphysik und Metapolitik. Studien zu Aristoteles und zur politischen Sprache der neuzeitlichen Philosophie, Suhrkamp, Frankfurt.

Rosenthal, F. (1932), Ibn Khalduns Gedanken über den Staat, R. Oldenbourg, München.

Rosenthal, E.I.J. (1958), Political Thought in Medieval Islam: An Introductory Outline, Cambridge University Press, Cambridge.

Said, E. W. (1978), Orientalism. Western Conceptions of the Orient, Pantheon Books, New York.

Sival, E. (1990), Radical Islam: Medieval Theology and Modern Politics, Yale University Press, New Haven. 
Suurland, D.A.J. (2009), "Totalitarianism and Radical Islamic Ideologies", en B.C. Labuschagne \& R.W. Sonnenschmidt, Religion, Politics and Law. Philosophical Reflections on the Sources of Normative Order in Society, Brill, Leiden.

Taji-Farouki , S. (ed.) (2006), Modern Muslim Intellectuals and the Qur'an, Oxford University Press, Oxford.

Tausch, A. -Heshmati, A. (2009), Asabiyya: Re-interpreting Value Change in Globalized Societies, Discussion Paper n. 4459, IZA, Bonn.

Toynbee, A. J. (1956), A Study of History, Oxford University Press, Oxford

Vivanco Saavedra, L. I. (2000), "Características esenciales del pensamiento historiográfico de Ibn Jaldún”, Opción. Revista de ciencias humanas y sociales, Año 16, n. 31.

Weber, M. (1904), Die Objektivität sozialwissenschaftlicher und sozialpolitischer Erkenntnis, J.C.B. Mohr, Tübingen.

Winkel, E. (1995), "Review of History of Islamic Philosophy by H. Corbin", Journal of Islamic Studies, n. 6.

Zelkina, A. (2011) "Book Review: Global Salafism. Islam's New Religious Movement", Religion, State and Society, vol. 39, Issue 2-3. 
